\title{
ON REDUCED ARCHIMEDEAN SKEW POWER SERIES RINGS
}

\author{
HAMED MOUSAVI, FARZAD PADASHNIK AND AYESHA ASLOOB QURESHI*
}

\begin{abstract}
In this paper, we prove that if $R$ is an Archimedean reduced ring and satisfy ACC on annihilators, then $R[[x]]$ is also an Archimedean reduced ring. More generally we prove that if $R$ is a right Archimedean ring satisfying the $A C C$ on annihilators and $\alpha$ is a rigid automorphism of $R$, then the skew power series ring $R[[x ; \alpha]]$ is right Archimedean reduced ring. We also provide some examples to justify the assumptions we made to obtain the required result.
\end{abstract}

\section{INTRODUCTION}

A ring $R$ is called left Archimedean, if for each nonunit element $r \in R$ we have $\bigcap_{n \in \mathbb{N}} r^{n} R=\{0\}$. The right Archimedean rings are defined in a similar way. In [16], it is shown that if $R$ is a domain and $\bigcap_{n \in \mathbb{N}} a^{n} R=0$, then the quotient field $Q(R[[x / a]])$ has infinite transcendent degree over the quotient field $Q(R[[x]])$. A very important result in this direction is that if $R$ is a domain (or is completely integrally closed) and satisfies $A C C P$, then $R$ is Archimedean, see $[1,16]$. However, each $A C C P$-domain is not necessarily Archimedean, see for example, [3, p. 1127]. There are similar results in [2, 4].

Ribenboim in [15] defined the ring of generalized power series $R[[S]$ consisting of all maps from $S$ to $R$ whose support is Artinian and narrow with the pointwise addition and the convolution multiplication. This construction provided interesting examples of rings, for example see $[13,14]$. In [12], Ribenboim gave a sufficient condition for the ring $R[[S]]$ (not necessarily commutative) to be Noetherian. However, Frohn gave an example to show that $A C C P$ does not rise to the power series ring in general (see [5]).

In [10], R. Mazurek and M. Ziembowski, introduced a "twisted" version of the Ribenboim construction and studied when it produces a von Neumann regular ring. They also proved that if $R$ is a domain and $\omega$ an endomorphism of $R$, then $R$ satisfies $A C C P L$, $S$ is an $A C C P L$-monoid and $\omega_{s}$ is injective for each $s \in S$ if and only if $R[[S, \omega]]$ is an $A C C P L$-domain.

As a similar result, for a strictly ordered monoid $S$ and a monoid homomorphism $\omega: S \rightarrow \operatorname{End}(R)$, in [11] it is proved that if the attached skew generalized power series ring $R[[S, \omega]]$ is a right Archimedean domain then $R$ is a right Archimedean domain, $S$ is a right Artinian and narrow monoid and $\omega_{s}$ is an injective which preserves nonunits of $R$ for any $s \in S$. Next step in this direction, we show that when $R$ is right (resp. left) Archimedean and satisfies the $A C C$ on annihilators, then $R[[x ; \alpha]]$ is a right (resp. left) Archimedean ring. In section 2, we give an example to justify that the condition

2010 Mathematics Subject Classification. 16P70, 16P60, 13F10, 13J05.

Key words and phrases. Skew power series ring, generalized power series rings, right (or left) Archimedean ring, Annihilator, Ascending chain conditions for principal one-sided ideals, ordered monoids. 
given in Theorem 2.2 is necessary on the ring $R$, to be able to pass the property of being Archimedean between $R$ and $R[[x ; \alpha]]$.

A ring $R$ is reduced, if the condition $a^{2}=0$ results in $a=0$. According to Krempa [9], an endomorphism $\alpha$ of a ring $R$ is said to be rigid if $a \alpha(a)=0$ implies $a=0$, for $a \in R$. A ring $R$ is said to be $\alpha$-rigid if there exists a rigid endomorphism $\alpha$ of $R$. Clearly, every domain $D$ with a monomorphism $\alpha$ is rigid. It is clear that, $\alpha$-rigid rings are reduced, (see e.g., [8]). The following Lemma by Hong in [8] is very useful in the following sections.

Lemma 1.1. Let $R$ be a $\alpha$-rigid ring and $a, b \in R$. Then

(1) $R$ is reduced.

(2) If $a b=0$, then we have $a \alpha^{n}(b)=\alpha^{n}(a) b=0$ for any positive integer $n$.

Proof. (1) Let $a^{2}=0$. Then $a \alpha(a) \alpha(a) \alpha^{2}(a)=0$. So $a \alpha(a) \alpha(a \alpha(a))=0$. Because $R$ is rigid $a \alpha(a)=0$. Another use of it gives us $a=0$.

(2) Let $a b=0$. So $b a=0$. This implies that $a \alpha^{n}(b a) \alpha^{2 n}(b)=0$. So $a \alpha^{n}(b) \alpha^{n}\left(a \alpha^{n}(b)\right)=$ 0 . Since $R$ is $\alpha$-rigid, then $a \alpha^{n}(b)=0$. Similarly $\alpha^{n}(a) b=0$.

Throughout this paper, all monoids and rings are with identity element that is inherited by submonoids and subrings and preserved under homomorphisms, but neither monoids nor rings are assumed to be - necessarily - commutative.

\section{ARCHIMEDEAN SKEW POWER SERIES RINGS}

In this section, we give the conditions on the ring $R$ such that the property of being Archimedean can be transferred from $R$ to the skew power series ring $R[[x ; \alpha]]$ and vice versa. The following results in [11, Theorems 4.1] describe such a transfer with the assumption that $R[[S, \omega]]$ is a domain.

Let $\omega: S \longrightarrow \operatorname{End}(R)$ be a monoid homomorphism, where $R$ is a $\operatorname{ring}$ and $(S, \leqslant)$ is a strictly ordered monoid. If $R[[S, \omega]]$ is a left (resp. right) Archimedean domain, then one can see that $R$ is a left (resp. right) Archimedean domain. It can be proved straightforward from the definition and the fact that $\bigcap_{n \in \mathbb{N}}\left(c_{a}\right)^{n} R[[S, \omega]]=0$ for nonunit a. Also, $S$ is a left (resp. right) Artinian and narrow monoid because of the fact that $\bigcap_{n \in \mathbb{N}}\left(\mathbf{e}_{s}\right)^{n} R[[S, \omega]]=0$ for nonunit $s \in S$. Moreover, $\omega_{s}$ is injective (and preserve nonunits of $R$ ) for any $s \in S$.

As mentioned in previous section, if $(S, ., \leqslant)$ is a (in particular positive) strictly totally ordered monoid and $0 \neq f \in R[[S, \omega]]$, then $\operatorname{supp}(f)$ is a nonempty well-ordered subset of $S$. The smallest element of $\operatorname{supp}(f)$ is denoted by $\pi(f)$ and we set $\theta(f):=f(\pi(f))$. In particular, for a power series $f(x)=\sum f_{n} x^{n}$, with the first nonzero coefficient is $f_{m}$, we have $\pi(f)=m$ and $\theta(f)=f_{m}$. Let $U(R)$ be the set of unit elements of $R$. Then it is shown in [10, Proposition 3.2] that if $\pi(f) \in U(S)$ and $\theta(f) \in U(R)$, then $f \in U(R[[S, \omega]])$, where $f \in R[[S, \omega]] \backslash\{0\}$.

Theorem 2.1. [11, Theorems 4.1] Let $R$ be a ring, $(S, \leqslant)$ a strictly ordered monoid and $\omega: S \longrightarrow \operatorname{End}(R)$ a monoid homomorphism. Then we have the following:

(i) The ring $R[[S, \omega]]$ is a right Archimedean domain if and only if $R$ is a right Archimedean domain, $\omega_{s}$ is injective and preserves nonunits of $R$ for any $s \in S$.

(ii) The ring $R[[S, \omega]]$ is a left Archimedean domain if and only if $R$ is a left Archimedean domain and $\omega_{s}$ is injective for any $s \in S$. 
Before stating the main result of the paper, we first recall a few definitions. An ideal $P$ of $R$ is completely prime if $a b \in P$ implies $a \in P$ or $b \in P$ for $a, b \in R$. Let $\alpha$ an endomorphism of $R$. An ideal $I$ of $R$ is called an $\alpha$-ideal if $\alpha(I) \subseteq I$ and $I$ is called $\alpha$-invariant if $\alpha^{-1}(I)=I$. Note that if $I$ is an $(\alpha)$-ideal, then $\bar{\alpha}: R / I \longrightarrow R / I$ defined by $\bar{\alpha}(a+I)=\alpha(a)+I$ for $a \in R$ is an endomorphism of the factor ring $R / I$.

We prove a similar result in the realm of reduced rings. Reduced rings are natural generalization of domains, for example direct product of domains is reduced. Therefore, Archimedean rings can also keep certain properties similar to the Archimedean domains.

Theorem 2.2. Let $R$ be a $\alpha$-rigid ring satisfying the $A C C$ on annihilators and $\alpha$ be a surjective endomorphism. Then:

(1) $R$ is right Archimedean and $\alpha$ preserves nonunits of $R$ if and only if the reduced ring $R[[x ; \alpha]]$ is right Archimedean .

(2) $R$ is left Archimedean if and only if the reduced ring $R[[x ; \alpha]]$ is left Archimedean.

Proof. We will give the proof for the right-sided version. The proof for the left-sided version follows on the same line if the order of the multiplication is changed from right to left throughout the proof. We recall that for a power series $g(x)=g_{0}+g_{1} x+\cdots$ we have $\pi(g):=m$ where $g_{m} \neq 0$ and $g_{i}=0$ for $0 \leqslant i<m$. Also $\theta(g)=g_{m}$ is the leading term of the power series. We set $\pi(0)=-\infty$.

First, assume that $R$ is right Archimedean. According to [6], if $R$ is rigid, then $R[[x ; \alpha]]$ is a reduced ring. Let $A=R[[x ; \alpha]]$ and

$$
\Gamma:=\left\{g \in A \mid g \text { is a nonunit in } A \text { and } \bigcap_{n \in \mathbb{N}} A g^{n} \neq\{0\}\right\} .
$$

We need to show that $\Gamma=\varnothing$. Assume that $\Gamma \neq \varnothing$. We define the set of leading terms $I_{f}:=\{\theta(g) \mid g \in f A\} \cup\{0\}$. Then, clearly $I_{f}$ is a right ideal of $R$. Let

$$
T:=\left\{\operatorname{Ann}\left(\bigcup_{i \in \mathbb{N}} I_{c_{i} g}\right) \mid c_{i} \in R, g \in \Gamma \text { and } c_{i} g \neq 0, c_{i} g^{i}=c_{j} g^{j} \forall i, j \in \mathbb{N}\right\} .
$$

Then $T \neq \varnothing$ because we assumed that $\Gamma \neq \varnothing$. Note that $\operatorname{Ann}\left(I_{c_{i} g}\right)=\operatorname{Ann}\left(I_{c_{i} g^{i}}\right)$ because $R$ is rigid. Considering that $R$ satisfies $A C C$ on annihilators, and then applying Zorn's Lemma results in the fact that $T$ has a maximal element. Let $V:=\operatorname{Ann}\left(\bigcup_{i \in \mathbb{N}} I_{a_{i} f}\right)$ be the maximal element of $T$ for some $f \in \Gamma$ such that

$$
a_{i} \in R, a_{i} f \neq 0 \text {, and } a_{i} f^{i}=a_{j} f^{j} \forall i, j \in \mathbb{N} \text {. }
$$

Step 1. We claim that $V$ is a two-sided completely prime ideal of $A$.

From Lemma 1.1, we see that $R$ is reduced because it is rigid. It implies that annihilators in $R$ are two-sided ideals. Let $a b \in V$ for some $a, b \in R$ and $b \notin V$. We need to show that $a \in V$. First, we show that $a \in \operatorname{Ann}\left(I_{b a_{i} f}\right)$ for all $i \in \mathbb{N}$. Observe that if $b a_{i} f h=0$ for all $h \in A$ and $i \in \mathbb{N}$, then $b \in V$ which contradicts our assumption that $b \notin V$. Therefore, $b a_{j} f A \neq\{0\}$ for some $j \in \mathbb{N}$. Take $b a_{j} f h \in b a_{j} f A$ such that $b a_{j} f h \neq 0$. This means that $\bigcup_{i} I_{b a_{i} f} \neq 0$. Also, for all $i \in \mathbb{N}$ and for all $h \in A$ we have $a b \theta\left(a_{i} f h b\right)=0$ because $a b \in V$ and $a_{i} f h b \in a_{i} f A$. Assume that $\theta\left(a_{i} f h b\right):=t b$, then $\theta\left(b a_{i} f h\right)=b t$ because $R$ is reduced. We conclude that $a b \theta\left(b a_{i} f h\right)=0$, because $R$ is reduced - since in every reduced ring $w y z=0$ implies $w z y=0$. 
Take $w=a$ and $y=b t$ and $z=b$. So $a b b t=a b^{2} t=0$. Again by using that $R$ is reduced, we get $a b t=0$. So $a \theta\left(b a_{i} f h\right)=0$ for all $i \in \mathbb{N}$ and $h \in A$; and we conclude that $a \in \operatorname{Ann}\left(\bigcup_{i \in \mathbb{N}} I_{b a_{i} f}\right)$.

Let $z \in V$. So $z \theta\left(a_{i} f l\right)=0$ for all $i>0$ and $l \in A$. In particular, $z \theta\left(a_{i} f h b\right)=0$ for all $h \in A$. Considering that $R$ is reduced, we get $z \theta\left(b a_{i} f h\right)=0$. This implies that $z \in \operatorname{Ann}\left(\bigcup_{i \in \mathbb{N}} I_{b a_{i} f}\right)$, which means that

$$
V \subseteq \operatorname{Ann}\left(\bigcup_{i \in \mathbb{N}} I_{b a_{i} f}\right)
$$

Also, since $b a_{i} f A \neq 0$, for some $i \in \mathbb{N}$ and $b a_{i} f^{i}=b a_{j} f^{j} \forall i, j \in \mathbb{N}$, we see that $\operatorname{Ann}\left(\bigcup_{i \in \mathbb{N}} I_{b a_{i} f}\right) \in T$. The equation (2.2) and the maximal property of $V$ in $T$ yields that $V=\operatorname{Ann}\left(\bigcup_{i \in \mathbb{N}} I_{b a_{i} f}\right)$. This implies that $a \in V$ and that $V$ is a two sided completely prime ideal.

Step 2. We show that $V$ is $\alpha$-invariant.

Let $r \in \alpha^{-1}(V)$. Then there exists $v \in V$ such that $\alpha^{-1}(v)=r$ and we have $\alpha(r)=v$. By definition of $V$, we have $\alpha(r) u=0$ for all $u \in \bigcup_{i \in \mathbb{N}}\left(I_{a_{i} f}\right)$. Then, from Lemma 1.1, we have $r u=0$ and it shows that $r \in V$ and $\alpha^{-1}(V) \subseteq V$.

To show the reverse inclusion, take $v \in V$. Then for all $u \in \bigcup_{i \in \mathbb{N}}\left(I_{a_{i} f}\right)$ we have $v u=0$. Again, by Lemma 1.1, we have $\alpha(v) u=0$. It shows that $\alpha(v) \in V$. Since $\alpha$ is surjective and rigid, hence an automorphism, we see that $v \in \alpha^{-1}(V)$ and therefore, $V \subseteq \alpha^{-1}(V)$. From this we conclude that $V$ is an $\alpha$-invariant.

Step 3. We show that $W:=\left(\frac{R}{V}\right)[[x ; \bar{\alpha}]]$ is a well-defined Archimedean domain.

We already know that $V$ is a two-sided completely prime ideal, so the factor $\operatorname{ring} U:=\frac{R}{V}$ is a well-defined domain. Then it is enough to show that $U$ is an Archimedean domain because then by using Theorem 2.1 we can conclude that $U[[x ; \bar{\alpha}]]$ is an Archimedean domain, where $\bar{\alpha}: R / V \longrightarrow R / V$ defined by $\bar{\alpha}(a+V)=\alpha(a)+V$ for all $a \in R$ is an endomorphism of factor ring $R / V$.

To show that $U$ is an Archimedean domain, we take $\bar{z} \in \bigcap_{i \in \mathbb{N}} U \bar{y}^{i}$. Then $\bar{z}=\overline{z_{1}} \bar{y}=$ $\overline{z_{2}} \bar{y}^{2}=\cdots$ for some $\overline{z_{i}} \in U$. It gives,

$$
z+l_{0}=z_{1} y+l_{1}=z_{2} y^{2}+l_{2}=\cdots
$$

for some $l_{i} \in V$. We choose $h \in A$ and $i \in \mathbb{N}$. By multiplying 2.3 with $\theta\left(a_{i} f h\right)$ and using that $l_{i} \in V$ for all $i$, we obtain $\theta\left(a_{i} f h\right) z=\theta\left(a_{i} f h\right) z_{1} y=\cdots$. Then $\theta\left(a_{i} f h\right) z=0$, because $R$ is Archimedean and $y$ is not unit in $R$, otherwise $\bar{y}$ becomes a unit in $U$. As $h$ and $i$ are chosen arbitrarily, we obtain that $z \in \operatorname{Ann}\left(I_{a_{i} f}\right)$ for all $i \in \mathbb{N}$ and therefore, $z \in V$. This shows, $\bar{z}=0$, as required. Therefore, $U$ is Archimedean.

Step 4. We claim that $\bar{f}$ is nonunit in $W=R / V[[x ; \bar{\alpha}]]$.

Assume that there exists $g \in A$ such that $\bar{f} \bar{g}-\overline{1}=\overline{0}$. It means that all coefficients of $f g-1$ belong to $V$. In particular, $\theta(f g-1) \in V$ and we have $\theta(f g-1) \theta\left(a_{i} f\right)=0$ for all $i \in \mathbb{N}$. Assume that $f(x)=\sum f_{l} x^{l}$ and $g(x)=\sum g_{l} x^{l}$. We show that $f_{0} g_{0}$ is not a unit.

Case 1. Assume that $\pi(f g-1)>0$. Then we have $f_{0} g_{0}=\theta(f g)=1$. Thus, $f_{0}$ is a unit. This implies that $f$ is a unit, which contradicts to our assumption that $f$ is non-unit. Therefore, we conclude that $\pi(f g-1)>0$ is not possible.

Case 2. Now, let $\pi(f g-1)=0$. Note that $(f g)_{0}-1 \in V$ and $(f g)_{0}-1 \neq 0,-1$, because otherwise it shows that $V=A$, which is impossible. Since $(f g)_{0} \neq 0$, then $(f g)_{0}$ 
is a nonunit, otherwise $f g$ is unit, [10, Proposition 3.2], which is not possible. Therefore, $f_{0} g_{0}$ is a nonunit.

We assumed that $\bar{f}$ is a unit, which implies that $\bar{f}^{i}$ is a unit for all $i \in \mathbb{N}$. For each $i \in \mathbb{N}$, let $\overline{b_{i}}$ be the inverse of $\bar{f}^{i}$. Then we have ${\overline{b_{i} f}}^{i}={\overline{b_{j} f}}^{j}=1$, for all $i, j \in \mathbb{N}$. It means that for all $i, j \in \mathbb{N}$ we have ${\overline{b_{i} f}}^{i}-{\overline{b_{j} f}}^{j}=0$ and all the coefficients of $b_{i} f^{i}-b_{j} f^{j}$ belong to $V$. It leads us to have $\theta\left(a_{1} f\right)\left(b_{i} f^{i}-b_{j} f^{j}\right)=0$ - because $R$ is reduced.

Therefore, we obtain

$$
\theta\left(a_{1} f\right)=\theta\left(a_{1} f\right) b_{1} f=\theta\left(a_{1} f\right) b_{2} f^{2}=\cdots .
$$

We know from equation (2.1) that $\theta\left(a_{1} f\right) \neq 0$, which gives $\theta\left(a_{1} f\right)\left(b_{i} f^{i}\right)_{0} \neq 0$. Let $b_{i}(x)=\sum b_{i, j} x^{j}$ and $t_{i}:=\theta\left(a_{1} f\right) b_{i, 0}$ and $r:=f_{0}$. One can see that $\left(b_{i} f^{i}\right)_{0}=b_{i, 0} f_{0}^{i}=b_{i, 0} r^{i}$. So $0 \neq \theta\left(a_{1} f\right)\left(b_{i} f^{i}\right)_{0}=t_{i} r^{i}$. Thus, $t_{i+1} r^{i+1}=t_{i} r^{i} \neq 0$ for all $i$. Note that $r \neq 0$ is a non-unit because $f$ is non-unit. It sums up to the result that $R$ is non-Archimedean which is contradiction to our hypothesis. Therefore, $\bar{f}$ must be a non-unit.

Step 5. Finally, we are ready to show that $A$ is an Archimedean reduced ring.

From equation (2.1), we have $\overline{a_{1}} \bar{f}=\overline{a_{2}} \overline{f^{2}}=\cdots$. In Step 3 and 4, we showed that $W$ is Archimedean domain and $\bar{f}$ is a non-unit in $W$, so we must have $\overline{a_{1}} \bar{f}=\overline{0}$ and consequently, $\overline{a_{1}}=\overline{0}$ or $\bar{f}=\overline{0}$. If $\overline{a_{1}}=\overline{0}$, then all coefficients of $a_{1}=\sum a_{1, j} x^{j}$ belong to $V$ and $a_{1, j} \theta\left(a_{1} f\right)=0$ for all $j \in \mathbb{N}$. Again, by using Lemma 1.1 we obtain

$$
\begin{aligned}
\left(\theta\left(a_{1} f\right)\right)^{2}=\left(\theta\left(a_{1} f\right)\right)\left(\theta\left(a_{1} f\right)\right) & =\theta\left(a_{1} f\right)\left(\sum_{x+y=\pi\left(a_{1} f\right)} a_{1, x} \alpha^{x}\left(f_{y}\right)\right) \\
& =\sum_{x+y=\pi\left(a_{1} f\right)}\left(\theta\left(a_{1} f\right) a_{1, x} \alpha^{x}\left(f_{y}\right)\right)
\end{aligned}
$$

Because $\theta\left(a_{1} f\right) a_{1, x}=0$ for all $x \in S$, we see that $\theta\left(a_{1} f\right)^{2}=0$. It gives that $\theta\left(a_{1} f\right)=0$ because $R$ is reduced. This gives us a contradiction to our assumption that $a_{i} f \neq 0$ for all $i \in \mathbb{N}$. Therefore, $\overline{a_{1}} \neq 0$. Then we must have $\bar{f}=\overline{0}$, that is, all coefficient of $f$ must belong to $V$ and $f_{j} \theta\left(a_{1} f\right)=0$ for all $j \in \mathbb{N}$. Then, from Lemma 1.1, we see that $\theta\left(a_{1} f\right) \alpha^{l}\left(f_{j}\right)=0$. This gives,

$$
\left(\theta\left(a_{1} f\right)\right)^{2}=\sum_{x+y=\pi\left(a_{1} f\right)} a_{1, x} \alpha^{x}\left(f_{y}\right) \theta\left(a_{1} f\right)=0
$$

which again implies that $\theta\left(\left(a_{1} f\right)\right)^{2}=0$ and contradicts our assumption that $a_{i} f \neq 0$ for all $i \in \mathbb{N}$. Therefore, our primary assumption $\Gamma \neq \varnothing$ is false. It shows that $A$ is an Archimedean ring, which completes the proof of the "only if" part.

Conversely, assume that $\bigcap_{n=1}^{\infty} R a^{n} \neq 0$ for some $a \in R$. Then $0 \neq \bigcap_{n=1}^{\infty} R a^{n} \subseteq$ $\bigcap_{n=1}^{\infty} A a^{n}$. But $A$ is a right Archemidean ring, so $a$ should be a unit in $A$. Thus, $f a=1$ for some $f \in A$. This means that $(f a)_{0}=1$. So we get $f_{0} a=1$. Hence $a$ is a unit in $R$, which leads us to the conclusion that $R$ is a right Archemidean ring.

We show that $\alpha$ preserves nonunits. If $b \in R$ is a nonunit element and $\alpha(b)$ is a unit, then $c \alpha(b)=1$ for some $c \in R$. Assume that $f(x) b=1$, then $f_{0} b=1$ which is not possible. So $b$ is not a unit in $A$. Also, $c^{n} x b^{n}=c^{n} \alpha\left(b^{n}\right) x=x=c^{m} x b^{m}$. So $x \in \bigcap_{n} A b^{n}$, while $b$ is not a unit in $A$. This contradiction shows that $\alpha(b)$ should not be a unit in $R$. 
In the case $\alpha=i d_{R}$ - but $R$ is still not necessarily commutative - we conclude the next corollary immediately.

Corollary 2.3. Let $R$ be a right (resp. left) Archimedean reduced ring which satisfies the $A C C$ on annihilators. Then the power series ring $R[[x]]$ is a right (resp. left) Archimedean reduced ring.

In Theorem 2.2, we proved that under some conditions on the ring $R$, it is possible to transfer the property of being Archimedean from $R$ to $R[[x ; \alpha]]$. It is natural to ask that what are the minimum conditions required on $R$ to allow such a transfer from $R$ to $R[[S, \omega]]$. With the help of the next example, we justify that the condition rigid required in Theorem 2.2 cannot be changed to reduced.

Example 2.4. Let $\mathbb{F}$ be any field and put $S:=(\mathbb{N} \cup\{0\}) \times(\mathbb{Z})$ with usual addition and usual order. Consider $\mathbb{F}[[S]]=\mathbb{F}[[(\mathbb{N} \cup\{0\}) \times(\mathbb{Z})]]:=T$. If $\mathbb{F}[[\mathbb{Z}]]:=R$, then one can see that $T=R[[\mathbb{N} \cup\{0\}]]:=R[[S]]$. Also, we can see that $R$ is isomorphic to the topological closure of $\mathbb{F}((x))$ where the metric is induced from the p-adic valuation $\operatorname{deg}(\cdot)$ and $x:=e_{(1,0)} \in T$ - we use the standard notation $e_{s}$ for $s \in S$ in the skew generalized power series rings.

Define $x:=e_{(1,0)} \in T$ and $y:=e_{(0,1)} \in T$. One can see that $T$ is an skew generalized power series rings of $R$ where $S$ is a positive stirctly totally ordered monoid. Then, $T$ is a Noetherian integral domain (i.e. a reduced ring). Also, $T$ satisfies both $A C C P$ and $A C C$ on annihilators. Take $\alpha: T \rightarrow T$ defined by

$$
\alpha(1)=1, \quad \alpha(x)=y^{-1}, \quad \alpha(y)=y .
$$

Note that $\alpha$ is not a monomorphism, and therefore, not rigid because $\alpha\left(x y^{2}-y\right)=0$ and $x y^{2}-y \neq 0$. So $T$ is not an $\alpha$-rigid ring.

Let $U:=T[[z ; \alpha]]$ and $f_{k}(z)=x \sum_{n=k}^{\infty} y^{n} z \in T$. Then

$$
f_{k+1}(z) \cdot x=\left(\sum_{n=k+1}^{\infty} x y^{n} z\right) x=\sum_{n=k+1}^{\infty} x \alpha(x) y^{n} z=\sum_{n=k+1}^{\infty} x y^{n-1} z=\sum_{n=k}^{\infty} x y^{n} z=f_{k}(z) .
$$

It shows that $f_{k} U \subseteq f_{k+1} U$ and we obtain an ascending chain of principal ideals in $T$. In order to show that $f_{k} U \subsetneq f_{k+1} U$, it is enough to show that $x$ is not unit in $U$. Suppose that $h(z) x=1$ for some $h(z) \in U$. Let $h(z)=\sum_{i=0}^{\infty} g_{i}\left(x, y, y^{-1}\right) z^{i}$, then $h(z) x=g_{0}\left(x, y, y^{-1}\right) x+\left(\sum_{i=1}^{\infty} g_{i}\left(x, y, y^{-1}\right) z^{i}\right) x=1$. It implies, $g_{0}\left(x, y, y^{-1}\right) x=1$ showing that $x$ is a unit in $T$, which is not true. Therefore, we obtain a non-stablizing chain in $U$ given by $f_{1} T \subsetneq f_{2} U \subsetneq \ldots$ Hence, $U$ does not satisfy $A C C P R$.

In [7], the authors give another example which also shows that the $\alpha$-rigid condition in Theorem 2.2 is not superfluous. A ring is $\alpha$-compatible if $a \alpha(b)=0$ results in $a b=0$ - see more information about $\alpha$-compatible rings in [6]. It is known that the set of $\alpha$-rigid rings are the intersection of the set of $\alpha$-compatible rings and reduced rings. We proved that Archimedean property can be shifted from the $\alpha$-rigid rings and propose a counterexample for the reduced rings. The remaining natural question which seeks an answer is:

Question 2.5. Let $R$ be an $\alpha$-compatible ring satisfying the $A C C$ on annihilators. Furthermore, let $\alpha$ be a surjective endomorphism in $R$. Then $R$ is right (resp. left) Archimedean if and only if the reduced ring $R[[x ; \alpha]]$ is right (resp. left) Archimedean. 


\section{REFERENCES}

[1] D. D. Anderson, B. G. Kang, and M. H. Park, Anti-Archimedean rings and power series rings, Comm. Algebra 26(10) (1998), 3223-3238.

[2] J. T. Arnold, Algebraic extensions of power series rings, Trans. Amer. Math. Soc. 267(1) (1981), $95-110$.

[3] T. Dumitrescu, S.O.I. Al-Salihi, N. Radu, T. Shah, Some factorization properties of composite domains $A+X B[X]$ and $A+X B[X]$, Comm. Algebra 28 (2000), 1125-1139.

[4] J. T. Condo, $L C M$-stability of power series extensions characterizes Dedekind domains, Proc. Amer. Math. Soc. 123(8) (1995), 2333-2341.

[5] D. Frohn, A counterexample concerning ACCP in power series rings, Comm. Algebra 30 (2002), 2961-2966.

[6] E. Hashemi and A. Moussavi, Polynomial extensions of quasi-Baer rings, Acta Math. Hungar. 107(3) (2005), 207-224.

[7] W. Heinzer and D. Lantz, ACCP in polynomial rings: a counterexample, Proc. Amer. Math. Soc. 121 (1994), 975-977.

[8] C.Y. Hong, N.K. Kim and T. Kwak, Ore extensions of Baer and p.p.-rings, J. Pure Appl. Algebra 151 (2000), 215-226.

[9] J. Krempa, Some examples of reduced rings, Algebra Colloq. 3(4) (1996), 289-300.

[10] R. Mazurek and M. Ziembowski, The ascending chain condition for principal left or right ideals of skew generalized power series rings, J. Algebra 322 (2009), 983-994.

[11] A. Moussavi, F. Padashnik, K. Paykan, Archemidean Skew Generalized Power Series Rings, Communications of the Korean Mathematical Society 34(2), (2019) 361-374.

[12] P. Ribenboim, Noetherian rings of generalized power series, J. Pure Appl. Algebra 79(3) (1992), 293-312.

[13] P. Ribenboim, Special properties of generalized power series, J. Algebra 173 (1995a), 566-586.

[14] P. Ribenboim, Some examples of valued fields, J. Algebra 173 (1995b), 668-678.

[15] P. Ribenboim, Semisimple rings and von Neumann regular rings of generalized power series, $J$. Algebra 198 (1997), 327-338.

[16] Ph. B. Sheldon, How changing $D[[X]]$ changes its quotient field, Trans. Amer. Math. Soc. 159 (1971), 223-244.

Farzad Padashnik, Department of Pure Mathematics, Faculty of Mathematical SciEnCes, Isfahan University, Isfahan, Iran.

E-mail address: f.padashnik1368@gmail.com

Hamed Mousavi, Mathematics Department, Georgia Institute of Technology, 686 Cherry Street NW, Atlanta, Georgia 30313, US.

E-mail address: hmousavi6@gatech.edu

Ayesha Asloob Qureshi, Sabanci University, Faculty of Engineering and Natural Sciences, Orta Mahalle, Tuzla 34956, Istanbul, Turkey

E-mail address: aqureshi@sabanciuniv.edu 